\title{
Soliton pulse propagation in the presence of disorder-induced multiple scattering in photonic crystal waveguides
}

\author{
Nishan Mann* and Stephen Hughes \\ Department of Physics, Queen's University, Kingston, Ontario, Canada, KYL 3N6
}

(Dated: November 10, 2018)

\begin{abstract}
We introduce a new coupled mode theory to model nonlinear Schrödinger equations for contrapropagating Bloch modes that include disorder-induced multiple scattering effects on nonlinear soliton propagation in photonic crystal waveguides. We also derive sub unit-cell coupling coefficients and use these to introduce a generalized length scale associated with each coupling effect. In particular, we define a multiple-scattering length scale that quantifies the spatial extent of a disorderinduced cavity mode. Our numerical simulations of nonlinear pulse propagation are in excellent qualitative agreement with recent experiments and provide insight into how disorder inhibits soliton propagation and other nonlinear propagation effects in photonic crystal waveguides.
\end{abstract}

PACS numbers: 42.70.Qs, 42.25.Fx, 42.82.Et, 42.81.Dp

Introduction. Slow light in photonic crystal waveguides (PCWs) can be exploited for enhancing nonlinear optical (NLO) interactions [1], and there has been considerable experimental progress with group velocities ranging between $c / 10$ to $c / 60$. Self phase modulation (SPM) in the presence of two photon absorption (2PA) and free carrier effects was observed by Monat et al. [2], while non-trivial scaling of SPM and three-photon absorption (3PA) was investigated by Husko et al. [3]. Colman et al. [4] utilized dispersion engineered PCWs to suppress 3PA which was critical in the demonstration of temporal pulse compression of higher order solitons. Other demonstrated NLO effects include third harmonic generation and highly efficient four wave mixing [5-7].

Despite these successes, one of the major limiting factors for exploiting NLO effects in PCWs is disorderinduced multiple scattering which roughly scales as $n_{g}^{2}$, where $n_{g}$ is the group index [8]. This limitation is somewhat suppressed through dispersion-engineering $[9,10]$ or by reducing the length of the PCW to less than 500 unit cells which lowers losses but typically increases the required pump power [2]. Regardless, in the slow light regime, coupling to disorder is unavoidable and any realistic model must include such effects. The theory of disorder on linear propagation in PCWs is well developed $[8,11-13]$. For modelling NLO effects in PCs, Bhat and Sipe [14] used multiple scales analysis and $k \cdot p$ theory to derive a dynamical nonlinear Schrödinger equation (NLSE), which is first-order in time, and their nonlinear coefficients use unit-cell averaged Bloch modes; however, the previously mentioned NLO works use the NLSE model adapted from the nonlinear fiber optics literature [15], where the NLSE is first-order in space and the nonlinear coefficients are generalized by a unit cell integration involving only the periodic part of the Bloch mode.

A severe shortcoming of current NLSEs applied to PCWs is their naive modelling of disorder-induced losses (if at all) as an effective loss parameter $\alpha$, which follows the Beer-Lambert law, known to breakdown in the regime of multiple scattering, arising from coupling between contra-propagating modes [16]. The effect of multiple scattering on soliton propagation is shown schematically in Fig. 1. Although some works have partly studied coupling between contra-propagating modes in the context of examining nonlinear bistability in finite periodic media $[17,18]$, it was for weak scattering and in the $a b$ sence of group velocity dispersion (GVD). For PCWs, neither of these assumptions holds true.

In this Letter, we introduce a powerful coupled-mode theory (CMT) to model two coupled NLSEs for contrapropagating Bloch modes, including the effects of GVD, disorder-induced multiple scattering, SPM and crossphase modulation (XPM). Unlike previous works, our nonlinear coupling coefficients are positional dependent because they involve an integration over the cross-section of the PCW. We also introduce a characteristic length scale corresponding to each coupling coefficient, including a length scale associated with multiple scattering, which denotes the spatial extent associated with a disorderinduced localized mode. Using the W1 PCW, we model solitons propagating in the presence of multiple scattering for several $n_{g}$ ranging from fast light to the slow light regime as shown in Fig. 2. When nonlinearities dominate over multiple scattering, the soliton's spectra shows a random fine peak structure, whereas when multiple scat-
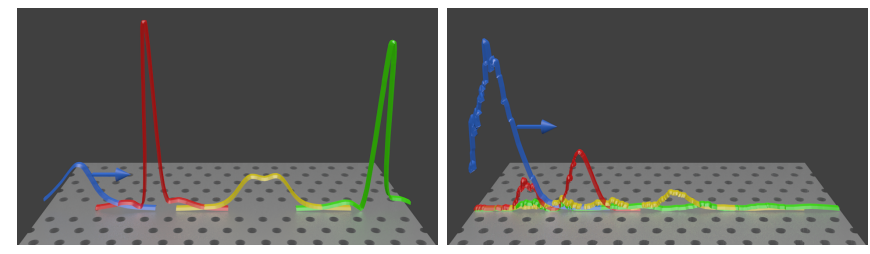

FIG. 1. (color online) Spatial profiles at various times of a soliton injected from the left side of a W1 PCW in the absence (left) and presence (right) of disorder-induced multiple scattering and localization. For comparing the two schematics, the two leftmost pulses (blue) are of the same magnitude. 


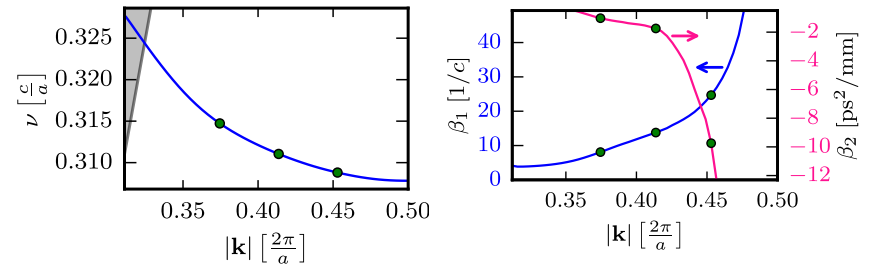

FIG. 2. (color online) Dispersion, group index $\left(\beta_{1}\right)$ and GVD $\left(\beta_{2}\right)$ characteristics of the W1 PCW with markers indicating the three values considered in this work.

tering dominates, the soliton's spectra exhibits narrow spectral peaks indicative of disorder-induced photon localization. Our numerical results are able to capture unexplained experimental features related to multiple scattering $[2,3,19]$ and our formalism can be generalized to assess the impact of multiple scattering on other nonlinearities such as 2PA, 3PA, self-steepening, four-wave mixing, etc.

Coupled NLSEs and coefficients. Denoting $x$ as the propagation direction, we rewrite Maxwell's equations as a Schrödinger-like equation in the frequency domain:

$$
A \psi=-i B \partial_{x} \psi
$$

where $A, B$ are Hermitian operators that contain the curl and divergence operations [20], and $\psi=\left[\mathbf{E}_{t} \mathbf{H}_{t}\right]^{T}$, where $\mathbf{E}_{t}, \mathbf{H}_{t}$ are the transverse components of electromagnetic fields. Since PCW possess discrete translational symmetry in $x, \psi$ has the Bloch mode form $\psi=e^{i k x} \varphi(x), \varphi(x+a)=\varphi(x)$, where $\varphi=\left[\mathcal{E}_{t} \mathcal{H}_{t}\right]^{T}$ and $\mathcal{E}, \mathcal{H}$ represent the periodic part of the Bloch mode. Using the Bloch mode form in (1) yields the generalized eigenvalue problem $C \varphi_{k}=k B \varphi_{k}, C=A+i \partial_{x} B$. Because $B$ is not positive-definite [21], one can derive the generalized orthogonality condition [22, 23],

$$
\left\langle\varphi_{k}, B \varphi_{k^{\prime}}\right\rangle=\delta_{k^{*}, k^{\prime}} \hat{\mathbf{x}} \cdot \int\left(\mathcal{E}_{t}^{\prime *} \times \mathcal{H}_{t}+\mathcal{E}_{t} \times \mathcal{H}_{t}^{*}\right) \cdot d \mathbf{a},
$$

where $k^{*}$ denotes the complex conjugate. For a TE-like guided mode as shown in Fig. 2, we have $\operatorname{Im}[k]=0$ (below the light line) so for $k=k^{\prime}$, one gets $\left\langle\varphi_{k}, B \varphi_{k}\right\rangle=4 \overline{S_{x}}$ where $\overline{S_{x}}$ denotes the $x$-component of the time-averaged Poynting vector. We now consider a dielectric perturbation to the ideal operator, $A^{0}$, as $A(x)=A^{0}+\Delta A(x)$, where $\Delta A(x)$ contains both linear and nonlinear perturbations, caused by disorder and the Kerr effect, respectively. The total wave function can be written as $\psi(x, \omega)=c^{+}(x, \omega) e^{i k x} \varphi_{k}(\omega)+c^{-}(x, \omega) e^{-i k x} \varphi_{-k}(\omega)$, where $c^{ \pm}(x, \omega)$ are envelope coefficients and $\varphi_{ \pm k}(\omega)$ are the unperturbed forward and backward eigenmodes; since multiple scattering between forward and backward modes is the major source of loss in PCWs, we include the small effect of out-of-plane scattering as an effective loss coefficient. To derive the coupled NLSEs for the field envelopes, we transform back to the time domain using the narrow bandwidth approximation $\Delta \omega / \omega \ll 1$, which also shifts zero frequency line to the centre frequency $\omega$, yielding:

$$
\begin{aligned}
& D^{+}\left[c^{+}\right]=i \frac{a \omega}{2 v_{g}}\left[Q_{(+,+)} c^{+}+Q_{(+,+)}^{|+|}\left|c^{+}\right|^{2} c^{+}+\left(Q_{(+,+)}^{|-|}+2 Q_{(+,-)}^{(+,-)}\right) 2\left|c^{-}\right|^{2} c^{+}+e^{-i 2 k x} Q_{(+,-)} c^{-}\right]-\frac{N}{2}\left\langle\alpha_{\mathrm{rad}}\right\rangle c^{+}, \\
& D^{-}\left[c^{-}\right]=-i \frac{a \omega}{2 v_{g}}\left[Q_{(-,-)} c^{-}+Q_{(-,-)}^{|-|}\left|c^{-}\right|^{2} c^{-}+\left(Q_{(-,-)}^{|+|}+2 Q_{(-,+)}^{(-,+)}\right) 2\left|c^{+}\right|^{2} c^{-}+e^{i 2 k x} Q_{(-,+)} c^{+}\right]+\frac{N}{2}\left\langle\alpha_{\mathrm{rad}}\right\rangle c^{-},
\end{aligned}
$$

with the coupling coefficients (related coefficients are obtained by just reversing the signs),

$$
\begin{aligned}
& Q_{(+,+)}=\int_{\mathbb{R}} \Delta \epsilon \mathcal{E}_{j}^{+*} \mathcal{E}_{j}^{+} d y d z, \quad Q_{(+,-)}=\int_{\mathbb{R}} \Delta \epsilon \mathcal{E}_{j}^{+*} \mathcal{E}_{j}^{-} d y d z, \quad Q_{(+,+)}^{|+|}=\int_{\mathbb{R}} \chi^{(3)}\left(\mathcal{E}_{l}^{+*} \mathcal{E}_{l}^{+}+2 \mathcal{E}_{j}^{+*} \mathcal{E}_{j}^{+}\right) \mathcal{E}_{j}^{+*} \mathcal{E}_{j}^{+} d y d z, \\
& Q_{(+,+)}^{|-|}=\int_{\mathbb{R}} \chi^{(3)} \mathcal{E}_{l}^{-*} \mathcal{E}_{l}^{-} \mathcal{E}_{j}^{+*} \mathcal{E}_{j}^{+} d y d z, \quad Q_{(+,-)}^{(+,-)}=\int_{\mathbb{R}} \chi^{(3)} \mathcal{E}_{j}^{+*} \mathcal{E}_{j}^{-} \mathcal{E}_{j}^{+*} \mathcal{E}_{j}^{-} d y d z
\end{aligned}
$$

where the Einstein summation convention is implied, $a$ is the lattice constant, $N$ is the number of unit cells, $D^{ \pm}:=\partial_{x} \pm \beta_{1} \partial_{t} \pm i \frac{\beta_{2}}{2} \partial_{t}^{2}, \omega$ is the centre frequency at which all subsequent quantities are defined, $\beta_{n}=$ $\left.\frac{d^{n} k}{d \omega^{\prime n}}\right|_{\omega^{\prime}=\omega}, \beta_{1} \equiv n_{g}$ and $v_{g}$ denotes the magnitude of the group velocity. The $j^{\text {th }}$ field component $(j=(x, y)$ for TE-like mode) is denoted by $\mathcal{E}_{j}^{ \pm}$but since we deal with forward and backward modes, they have the special property $\mathcal{E}_{j}^{-}=\mathcal{E}_{j}^{+*} ;\left\langle\alpha_{\mathrm{rad}}\right\rangle$ denotes the incoherent radiation loss per unit cell which accounts for out-of-plane scattering [16], and $\Delta \varepsilon$ represents the disorder caused by stochastic radial fluctuations of the etched air holes which are characterized by their rms roughness $\sigma$ and correlation length $l_{c}$-which is a measure of how strongly two intrahole fluctuations are correlated $[8,24,25]$ - see supplementary information (SI).

We assume that $\chi^{(3)}$ is isotropic with a nonlinear Kerrlike electronic response and is piecewise constant defined as non-zero in the slab only and vanishing in the air holes. The nonlinear scattering terms involving $e^{ \pm i 2 k x}$ were ne- 
glected because of the large phase mismatch $\Delta k=2 k$. One can show this assumption remains valid as long as the inequality $k L_{W 1} \gg \pi$ is satisfied where $L_{W 1}$ denotes the PCW length [26]. Given $k$ values shown in Fig. 2, one obtains the lower bound $L_{W 1} \gg 2 a$; note this does not imply that the stochastic linear scattering terms $Q_{(+,-)}, Q_{(-,+)}$are also negligible [22].

Treating $x(t)$ as the time (space) variables, (3)-(4) use the unusual initial conditions $c^{+}(0, t) \neq 0, c^{-}\left(L_{W 1}, t\right)=0$ unique to contra-propagating modes, where $L_{W 1} \equiv N a$ and we choose periodic boundary conditions $c^{ \pm}(x, t+$ $T)=c^{ \pm}(x, t)$ to avoid numerical reflections. Without radiation loss, our equations satisfy the power conservation law $\partial_{x}\left(\left\|c^{+}\right\|^{2}-\left\|c^{-}\right\|^{2}\right)=0 \quad[27]$, where $\left\|c^{ \pm}(x)\right\|^{2}:=$ $\int_{\mathbb{R}}\left|c^{ \pm}(x, t)\right|^{2} d t$, which states that the net power flow through any cross section of the PCW is conserved such that $\int_{\mathbb{R}}(T+R) d t=\left\|c^{+}(0)\right\|^{2}$ where the transmission and reflection of pulses is defined as $T=\left|c^{+}\left(L_{W 1}\right)\right|^{2}, R=$ $\left|c^{-}(0)\right|^{2}$, respectively. Lastly, if we turn off nonlinearities, (3)-(4) recover previous linear equations [13].

Field renormalization and characteristic length scales. Often CM equations are presented for mode envelopes, renormalized to have dimension of power, i.e. $C^{ \pm}=$ $\frac{2 v_{g} \epsilon_{0} U_{\mathbf{E}}}{a} c^{ \pm}$, where $\epsilon_{0}$ is the free space permitivitty and $U_{\mathbf{E}}=\int_{\text {cell }} \epsilon(\mathbf{r})\left|\mathbf{E}_{\mathbf{k}}\right|^{2} d \mathbf{r}$ is the Bloch mode energy. If (3)(4) are renormalized it causes the nonlinear terms involving $\left|C^{ \pm}\right|^{m-1}$ ( $m$ represents the odd order of the nonlinearity) to scale as scale $v_{g}^{-(m-1)}[3,4,14]$, while the linear terms retain their $v_{g}^{-1}$ scaling. In (3)-(4), however, we do not perform this renormalization, hence each term (linear or nonlinear) scales with the same factor of $v_{g}^{-1}$. Recently, Colman showed that this approach is much better suited for numerical calculations [28].

We choose the field normalization $U_{\mathbf{E}}=1$, such that $U_{\mathbf{E}}$ becomes dimensionless, $\left[U_{\mathbf{E}}\right]=1$; thus the electric field has dimensions $[\mathbf{E}]=1 / L^{\frac{3}{2}}$ and the coupling coefficients have dimension $1 / L$. Conveniently, we can use the inner product norm to define a characteristic length scale associated with each coupling coefficient, e.g., the SPM term $Q_{(+,+)}^{|+|}(x)$ has an associated length scale

$$
L_{Q_{(+,+)}^{|+|}}=\left[\frac{1}{L_{W 1}} \int_{0}^{L_{W 1}}\left|Q_{(+,+)}^{|+|}(x)\right|^{2} d x\right]^{-\frac{1}{2}}
$$

which is a generalization of the SPM length scale defined by Agrawal [15]. The length scale for GVD is defined as $L_{\beta_{2}}=T_{0}^{2} / \beta_{2}$ where $T_{0}$ is the pulse width. Finally, $\chi^{(3)}$ has dimensions $L^{3}$ but since the nonlinear susceptibility is usually in SI units $\left(\chi_{\mathrm{SI}}^{(3)}\right)[26]$, we use the conversion $\chi^{(3)}=\frac{n_{0} a P}{2 \epsilon_{0} c} \chi_{\mathrm{SI}}^{(3)}$, where $n_{0}$ is the refractive index of the slab and $P$ the incident peak power.

Modelling soliton propagation with disorder. As a concrete application of our theory, we numerically study soliton propagation in disordered PCWs. We consider a W1 PCW with $a=480 \mathrm{~nm}, r=0.2 a, h=0.333 a$, where
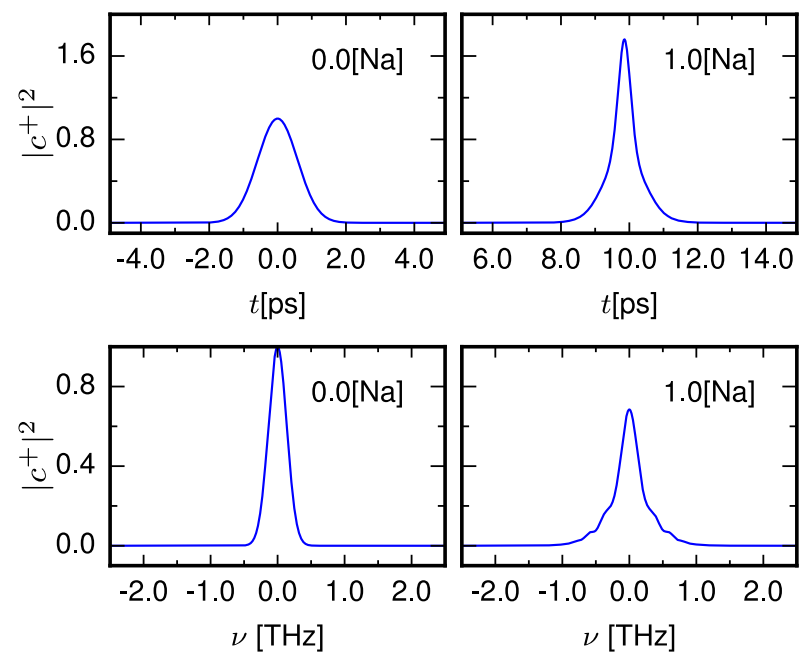

FIG. 3. (color online) Unchirped Gaussian pulse propagating in a W1 with $\beta_{1}=24.69, \beta_{2}=-9.7440 \mathrm{ps}^{2} / \mathrm{mm}, S=2.4$ and no disorder. Top: temporal snapshots in space taken at the beginning $(x=0)$ and end of the PCW $(x=1)$ showing temporal pulse compression. Bottom: spectral snapshots showing slight spectral broadening and fine structure characteristic of large anomalous GVD.
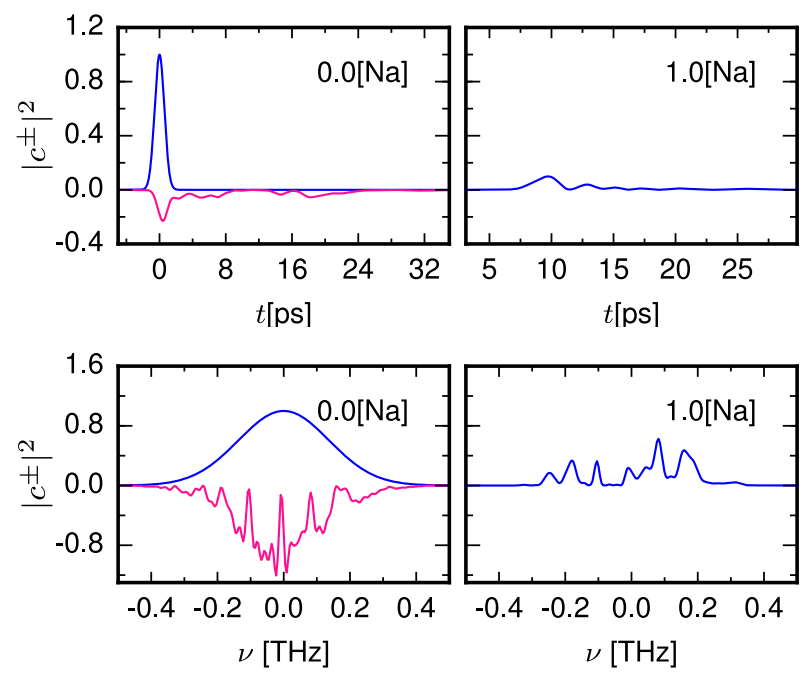

FIG. 4. (color online) Using the same dispersion parameters and initial conditions as in Fig. 3, but now in the presence of disorder-induced multiple scattering with rms roughness $\sigma=0.017 a$. The rise of a backwards travelling pulse due to multiple scattering is also shown (pink/light). Top: temporal snapshots showing pulse degradation. Bottom: spectral snapshots showing strong backreflection and weak transmission.

$a, r, h$ represent the pitch, hole radius and slab thickness, respectively. We fix the number of unit cells at $N=251$ which corresponds to a waveguide length of $L_{W 1}=120.48 \mu \mathrm{m}$. For initial conditions, we specify a forward propagating unchirped Gaussian pulse at one end of the PCW with zero backward pulse at the other end as $c^{+}(0, t)=e^{-t^{2} / 2 T_{0}^{2}}, c^{-}\left(L_{W 1}, t\right)=0$. The soliton number $S$ 

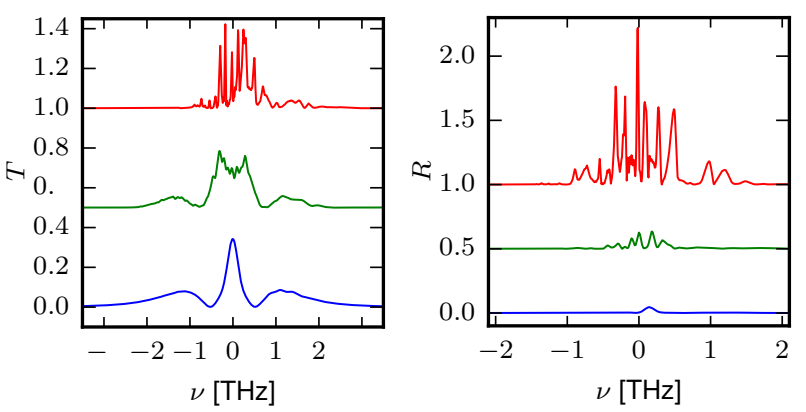

FIG. 5. (color online) Transmission (left) and reflection (right) spectra of an unchirped Gaussian pulse propagating in the presence of coupling to the contra-propagating mode via multiple scattering, SPM and XPM, for three different group indices: 8.093(blue-bottom), 13.782(green-middle) and 24.69(red-top). The PCW is $251 a$ unit cells long with rms roughness fixed at $0.017 a$.
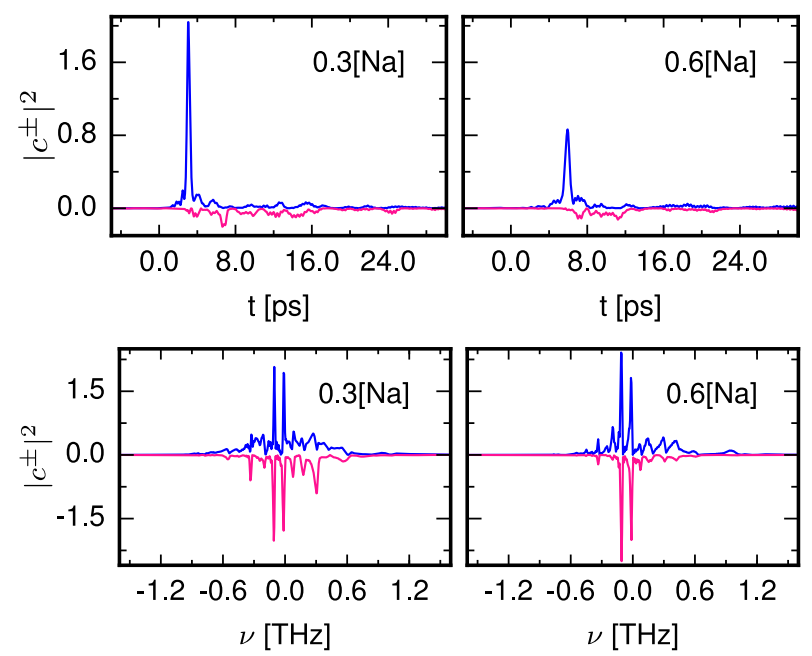

FIG. 6. (color online) For $\beta_{1}=24.69$ temporal and spectral profiles for the forward (blue/dark-solid) and the backward (pink/light-solid) mode envelopes at two different points inside the PCW. Strong multiple scattering dominates over SPM/XPM effects leading to sharp spectral peaks corresponding to the formation of localized modes.

is defined as $S^{2}=L_{\beta_{2}} / L_{Q_{+,+1}^{|+|}}[15]$, and we fix the incident peak power $P$ to study higher order solitons $(S>1)$ exclusively. The nonlinear coefficient is chosen to approximate a GaInP slab, $\chi_{\mathrm{SI}}^{(3)}=3 \times 10^{-19} \mathrm{~m}^{2} / \mathrm{V}^{2}$ [4]. We fix the correlation length at $0.083 a$ [24], and to observe multiple scattering effects with 251 unit cells, we choose the rms roughness $\sigma=0.017 a$. For the remainder of our discussion, as shown in Fig. 2, we choose three group indices $\beta_{1}=8.093,13.78,24.69$ having soliton numbers $S=4.6,4.1,2.4$, respectively.

To solve (3)-(4) numerically, we implement an implicit finite-difference scheme that steps forward in $x$ (the details will be described elsewhere). We first examine temporal pulse compression of a soliton propagating in the absence of disorder, hence no coupling to the backwards mode for $\beta_{1}=24.69, S=2.4$. The squared amplitudes of the mode envelopes $\left|c^{ \pm}(x, t / \omega)\right|^{2}$ at the opposite ends of the $\mathrm{W} 1$ are plotted in Fig. 3. In agreement with recent experiments [4], temporal pulse compression is clearly visible in the time domain at $x=1$ along with a spectrally broadened peak with fine structure characteristic of anomalous GVD. Next, we turn off nonlinearities and study only the effect of multiple scattering on pulse propagation as shown in Fig. 4 for the same dispersion parameters. In the time domain, it is seen that multiple scattering distorts the trailing edge of the incident pulse $(x=1)$ while simultaneously giving rise to a backwards wave $(x=0)$. In the spectral domain, the large backscattered signal and the emergence of spectral peaks in the transmission signals the onset of disorder-induced photon localization [13].

With our NLSEs, we can now model the combined effects of GVD, multiple scattering, SPM and XPM, as shown in Figs. 5, 6. Fig. 5 shows the transmission and reflection spectrums for all three group indices which are readily measured in experiments. As expected, for fast light $\beta_{1}=8.093$, the effect of multiple scattering is barely visible as the transmitted spectrum is dominated by nonlinear spectral broadening and minimal reflection. As we the increase the group index to $\beta_{1}=13.782$, the effect of multiple scattering begins to manifest in the spectral domain via the generation of a fine peak structure that slightly distorts the transmission spectrum whilst giving rise to a non-negligible reflection. This is in very good agreement with related experimental spectra obtained for similar group indices $[2,3]$. For $\beta_{1}=24.69$, which lies in the slow light regime, the transmission is greatly reduced and reflection enhanced as disorderinduced scattering now dominates over the Kerr nonlinearity as one sees large pulse distortion in the spectral domain. Fig. 6 shows the temporal and spectral pulse profiles for $\beta_{1}=24.69$ at two different spatial positions inside the PCW which can be measured using nondestructive experimental methods such as NSOM [19]. Temporally, one sees the compressed pulse being distorted by multiple scattering which manifests as sharp spectral resonances which are roughly of the same magnitude for both the forward and backward propagating modes and so in accordance with the power conservation law, this signals the formation of weakly localized states formed via multiple scattering inside the PCW. The pulse profiles for $\beta_{1}=13.782$ and increased $\mathrm{rms}$ roughness $\sigma=0.025 a$ are shown in the SI.

Multiple scattering length scale. While our definition for SPM/XPM length scales follow the standard interpretation [15], we also introduce here, $L_{Q_{(+,+)}}$and $L_{Q_{(+,-)}}$ as the characteristic linear SPM and multiple scattering length scales, respectively. From an ensemble of 100 disorder instances, we have calculated both the mean and standard deviation of $L_{Q_{(+,+)}}, L_{Q_{(+,-)}}$for various $\sigma$ 
$(0.008 a-0.025 a)$ and $n_{g}$. For all cases, particularly in the slow light regime, we find the standard deviation of these length scales to be of the order of a unit cell $\mathcal{O}(1 a)$ which is negligible so an instance value of these length scales is an excellent approximation of their expected value. We interpret the multiple scattering length scale to be a measure of the spatial extent of a disorder-induced cavity mode, e.g., near the mode-edge for $\sigma=0.008 a$, with $\beta_{1}=24.69$, we compute $L_{Q_{(+,-)}} \approx 10 a$ which is in good agreement with 3D FDTD simulations [25]. Recent experimental observations of localized modes in the presence of intrinsic disorder by Faggiani et al. [29] show the spatial extent of the localized modes to be roughly $15 a$ for similar values of disorder and group indices. Moreover, recent work by Xue et al. [30] on examining threshold characteristics of PC cavity lasers have found that the threshold gain attains a minimum for a cavity length of around $10 a$ which they attribute to disorder.

Conclusions. We have introduced coupled NLSEs for envelopes of contra-propagating modes in PCWs that include the effects of anomalous GVD, SPM, XPM and, most importantly, disorder-induced multiple scattering. We are also able to provide an elegantly simple definition of the characteristic length scales associated with each effect. Our results demonstrate the importance of multiple scattering on soliton propagation, and as an application we have qualitatively reproduced the fine peak structure of recent experimental spectra and we have predicted new features that can be accessed experimentally. Our theory can be extended to model a wide range of NLO effects.

We sincerely thank Pierre Colman for insightful discussions and NSERC for funding.

* nishan.mann@queensu.ca

[1] T. F. Krauss, Nat. Photonics 2, 448 (2008).

[2] C. Monat, B. Corcoran, M. Ebnali-Heidari, C. Grillet, B. J. Eggleton, T. P. White, L. O'Faolain, and T. F. Krauss, Opt. Express 17, 2944 (2009).

[3] C. Husko, S. Combrié, Q. V. Tran, F. Raineri, C. W. Wong, and A. De Rossi, Opt. Express 17, 22442 (2009).

[4] P. Colman, C. Husko, S. Combrié, I. Sagnes, C. W. Wong, and A. De Rossi, Nat. Photonics 4, 862 (2010).

[5] C. Monat, M. Spurny, C. Grillet, L. O'Faolain, T. F. Krauss, B. J. Eggleton, D. Bulla, S. Madden, and B. Luther-Davies, Opt. Lett. 36, 2818 (2011).

[6] C. Xiong, C. Monat, A. S. Clark, C. Grillet, G. D. Marshall, M. J. Steel, J. Li, L. O'Faolain, T. F. Krauss, J. G. Rarity, and B. J. Eggleton, Opt. Lett. 36, 3413 (2011).
[7] J. Li, L. O'Faolain, and T. F. Krauss, Opt. Express 20, 17474 (2012).

[8] S. Hughes, L. Ramunno, J. F. Young, and J. E. Sipe, Phys. Rev. Lett. 94, 033903 (2005).

[9] L. O'Faolain, S. A. Schulz, D. M. Beggs, T. P. White, M. Spasenović, L. Kuipers, F. Morichetti, A. Melloni, S. Mazoyer, J. P. Hugonin, P. Lalanne, and T. F. Krauss, Opt. Express 18, 27627 (2010).

[10] N. Mann, S. Combrié, P. Colman, M. Patterson, A. De Rossi, and S. Hughes, Opt. Lett. 38, 4244 (2013).

[11] B. Wang, S. Mazoyer, J. P. Hugonin, and P. Lalanne, Phys. Rev. B - Condens. Matter Mater. Phys. 78, 245108 (2008).

[12] S. Mazoyer, J. P. Hugonin, and P. Lalanne, Phys. Rev. Lett. 103, 063903 (2009).

[13] M. Patterson, S. Hughes, S. Combrié, N. V. Q. Tran, A. De Rossi, R. Gabet, and Y. Jaouën, Phys. Rev. Lett. 102, 253903 (2009).

[14] N. A. R. Bhat and J. E. Sipe, Phys. Rev. E 64, 56604 (2001).

[15] G. P. Agrawal, Nonlinear Fiber Optics, 4th ed., Electronics \& Electrical (Academic Press, 2007).

[16] M. Patterson, S. Hughes, S. Schulz, D. M. Beggs, T. P. White, L. O'Faolain, and T. F. Krauss, Phys. Rev. B 80, 195305 (2009).

[17] H. G. Winful and C. G. D., Appl. Phys. Lett. 40, 298 (1982).

[18] C. Martijn de Sterke and J. E. Sipe, Phys. Rev. A 42, 2858 (1990).

[19] C. Husko, M. Wulf, S. Lefrancois, S. Combrié, G. Lehoucq, A. De Rossi, B. J. Eggleton, and L. Kuipers, Nat. Commun. 7, 11332 (2016).

[20] S. Johnson, M. Ibanescu, M. Skorobogatiy, O. Weisberg, T. Engeness, M. Soljacic, S. Jacobs, J. Joannopoulos, and Y. Fink, Opt. Express 9, 748 (2001).

[21] S. Johnson, P. Bienstman, M. A. Skorobogatiy, M. Ibanescu, E. Lidorikis, and J. D. Joannopoulos, Phys. Rev. E 66, 066608 (2002).

[22] D. Marcuse, Theory of Dielectric Optical Waveguides, 1st ed. (Academic Press, New York, 1974) p. 257.

[23] D. Michaelis, U. Peschel, C. Wächter, and A. Bräuer, Phys. Rev. E 68, 065601 (2003).

[24] N. Mann, M. Patterson, and S. Hughes, Phys. Rev. B 91, 245151 (2015).

[25] N. Mann, A. Javadi, P. D. García, P. Lodahl, and S. Hughes, Phys. Rev. A 92, 023849 (2015).

[26] R. W. Boyd, Nonlinear Optics, 3rd ed. (Academic Press, 2008) p. 640.

[27] B. Reichel and S. Leble, Comput. Math. with Appl. 55, 745 (2008).

[28] P. Colman, Phys. Rev. A 92, 013827 (2015).

[29] R. Faggiani, A. Baron, X. Zang, L. Lalouat, S. A. Schulz, B. O'Regan, K. Vynck, B. Cluzel, F. de Fornel, T. F. Krauss, and P. Lalanne, Sci. Rep. 6, 27037 (2016).

[30] W. Xue, Y. Yu, L. Ottaviano, Y. Chen, E. Semenova, K. Yvind, and J. Mork, Phys. Rev. Lett. 116, 063901 (2016). 


\title{
Soliton pulse propagation in the presence of disorder-induced multiple scattering in photonic crystal waveguides: Supplementary Information
}

\author{
Nishan Mann* and Stephen Hughes \\ Department of Physics, Queen's University, Kingston, Ontario, Canada, K' 7 L 36
}

(Dated: November 10, 2018)

\begin{abstract}
Here we supply supplementary material that accompanies the manuscript "Soliton pulse propagation in the presence of disorder-induced multiple scattering in photonic crystal waveguides." We first explain the mathematical link between the three parameters characterizing disorder in photonic crystal waveguides; the dielectric perturbation, rms roughness and correlation length. We also provide two additional figures that supplement Figs. 5,6 in the Letter by showing pulse profiles inside the waveguide for $\beta_{1}=13.782$ and in the presence of increased disorder characterized by rms roughness $\sigma=0.025 a$.
\end{abstract}

The nonlinear coupling coefficients and the associated length scales defined in the main Letter can be computed once the ideal Bloch modes, nonlinear susceptibility and the incident peak power are known, but to compute the linear coefficients associated with disorder $Q_{(+,+)}, Q_{(-,-)}, Q_{(+,-)}, Q_{(-,+)}$, one requires information about the dielectric perturbation $\Delta \epsilon$. The dielectric perturbation caused by disorder is shown schematically in Fig. S1. Intrinsic fabrication disorder in photonic crystal waveguides (PCWs) refers mainly to the deviation of the etched air holes from their ideal cylindrical shape as shown in Fig. S1(a). Given state-of-the-art manufacturing techniques, a common approximation for photonic crystal (PC) slabs with etched holes is to assume the deviated cross section is constant throughout the cylinders height, hence one only needs to address in-plane variations which we model via the standard deviation of the rapid radial fluctuations $\sigma$ and a correlation length $l_{c}$-which in this case is a measure of how strongly two intrahole fluctuations are correlated (see Fig. S1(b)).

(a)

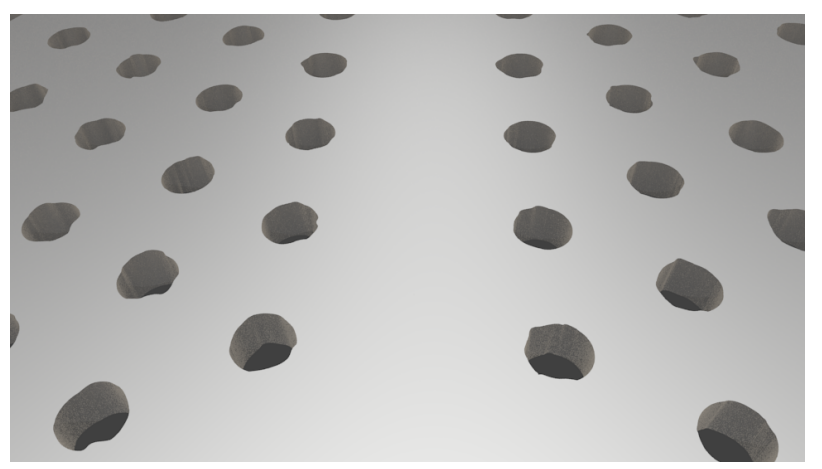

(b)

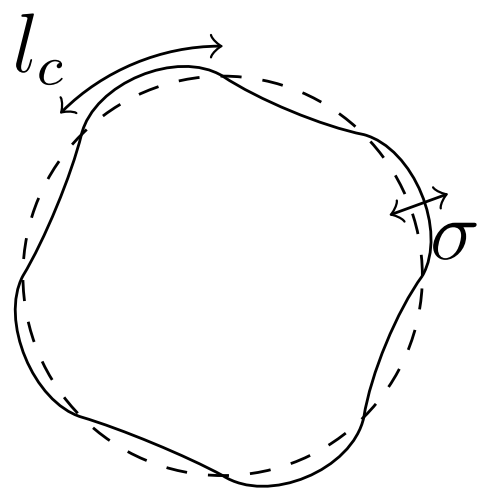

FIG. S1. (a) Schematially illustrating manufacturing imperfections in a PCW. (b) Schematic showing an ideal cross section of a cylindrical hole (dashed) along with the cross section of a disordered hole (solid). The labels illustrate the statistical parameters used for modelling surface roughness namely rms roughness $\sigma$ and correlation length $l_{c}$.

Using $i$ to index holes in the $\mathrm{PCW}, \Delta \epsilon$ in cylindrical coordinates is expressed as

$$
\Delta \epsilon=\left(\epsilon_{a}-\epsilon_{s}\right) \mathbb{1}_{\mathrm{PCW}} \sum_{i} \Delta r\left(\theta_{i}\right) \delta\left(r-r_{i}\right),
$$

where $\epsilon_{a / s}$ denotes the dielectric constant of air and PC slab respectively, $\mathbb{1}_{\mathrm{PCW}}$ is the indicator function (1 in the PCW, 0 otherwise), $\Delta r\left(\theta_{i}\right)$ denotes the radial perturbation at the azimuthal angle $\theta_{i}, r_{i}$ denotes the radius of the $i^{t h}$ hole and $\delta\left(r-r_{i}\right)$ is the Dirac-Delta function indicating that the disorder can be approximated as occurring only near the circumference of the $i^{t h}$ hole. Since disorder is a stochastic process, we assume $\mathbb{E}\left[\Delta r\left(\theta_{i}\right)\right]=0[$ ? ] and the

\footnotetext{
*nishan.mann@queensu.ca
} 
covariance between two radial perturbations $\Delta r\left(\theta_{i}\right), \Delta r\left(\theta_{j}^{\prime}\right)$ is given by [? ]

$$
\operatorname{cov}\left(\Delta r\left(\theta_{i}\right), \Delta r\left(\theta_{j}^{\prime}\right)\right)=\sigma^{2} e^{-r_{i}\left|\theta_{i}-\theta_{j}^{\prime}\right| / l_{c}} \delta_{i j}
$$

Using (S1) and (S2), we numerically generate radial perturbations for each hole which are then used to compute the integral expressions of the coupling coefficients associated with disorder.

In Fig. 6 of the Letter, we show the squared amplitudes of the mode envelopes $\left|c^{ \pm}\right|^{2}$ in both time and frequency domains at two spatial points within the W1 PCW, namely at $x=0.3,0.6$ for $\beta_{1}=24.69$ which lies in the slow light regime. In that figure, we see how disorder-induced multiple scattering severely distorts the pulse profile by forming localized modes. In Fig. S2, we show the profile for $\beta_{1}=13.782$, a value that lies between fast and slow light where multiple scattering slightly distorts the pulse profiles profiles inside the PCW. This is more easily seen in the frequency domain because in the time domain, the distortion amounts to fine ripples introduced in the tail of the compressed pulses $(t>2 \mathrm{ps})$ which can be measured experimentally by using non-destructive techniques such as NSOM.
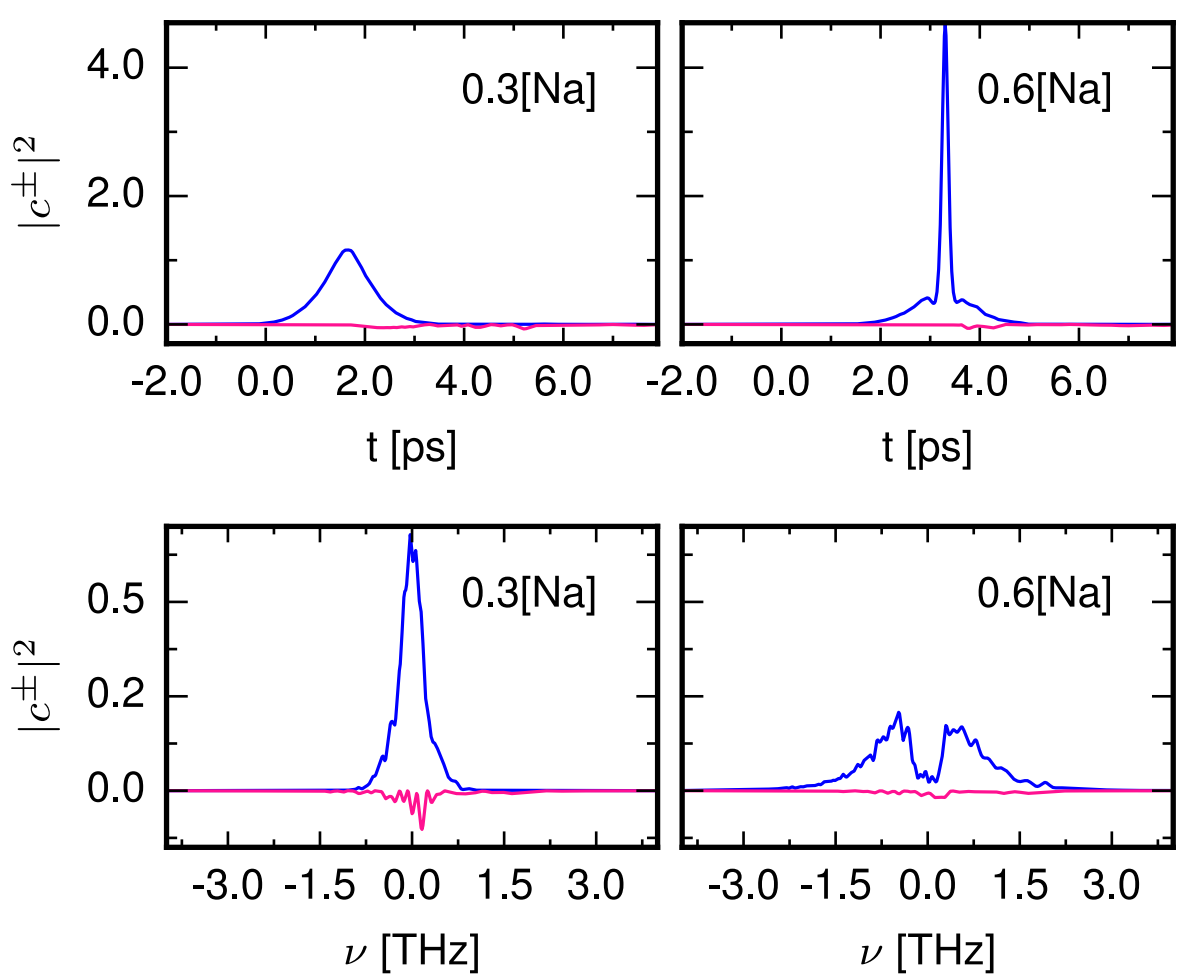

FIG. S2. (color online) For $\beta_{1}=13.782$ temporal and spectral profiles for the forward (blue/dark-solid) and the backward (pink/light-solid) mode envelopes at two different points inside the PCW. In this case, multiple scattering slightly distorts temporal pulse compression and spectral broadening caused by the Kerr nonlinearity.

We next increase the rms roughness to $\sigma=0.025 a$ to examine transmission, reflection and pulse profiles inside the W1 PCW in the presence of enhanced multiple scattering in Fig. S3. As the transmission spectrums show, for fast-light $\left(\beta_{1}=8.093\right)$, the increased disorder has little effect but as we move towards the slow light regime, enhanced multiple scattering causes more distortions particularly in the transmission spectrum of $\beta_{1}=13.782$ as the spectral pulse broadening shown in Fig. 5 of the Letter has been hampered. When looking at temporal pulse profiles inside the W1 PCW, it is now much clearer that distortions start to occur from the tail end of the pulse and slowly creep up to the peak of the pulse eventually breaking up the envelope. 

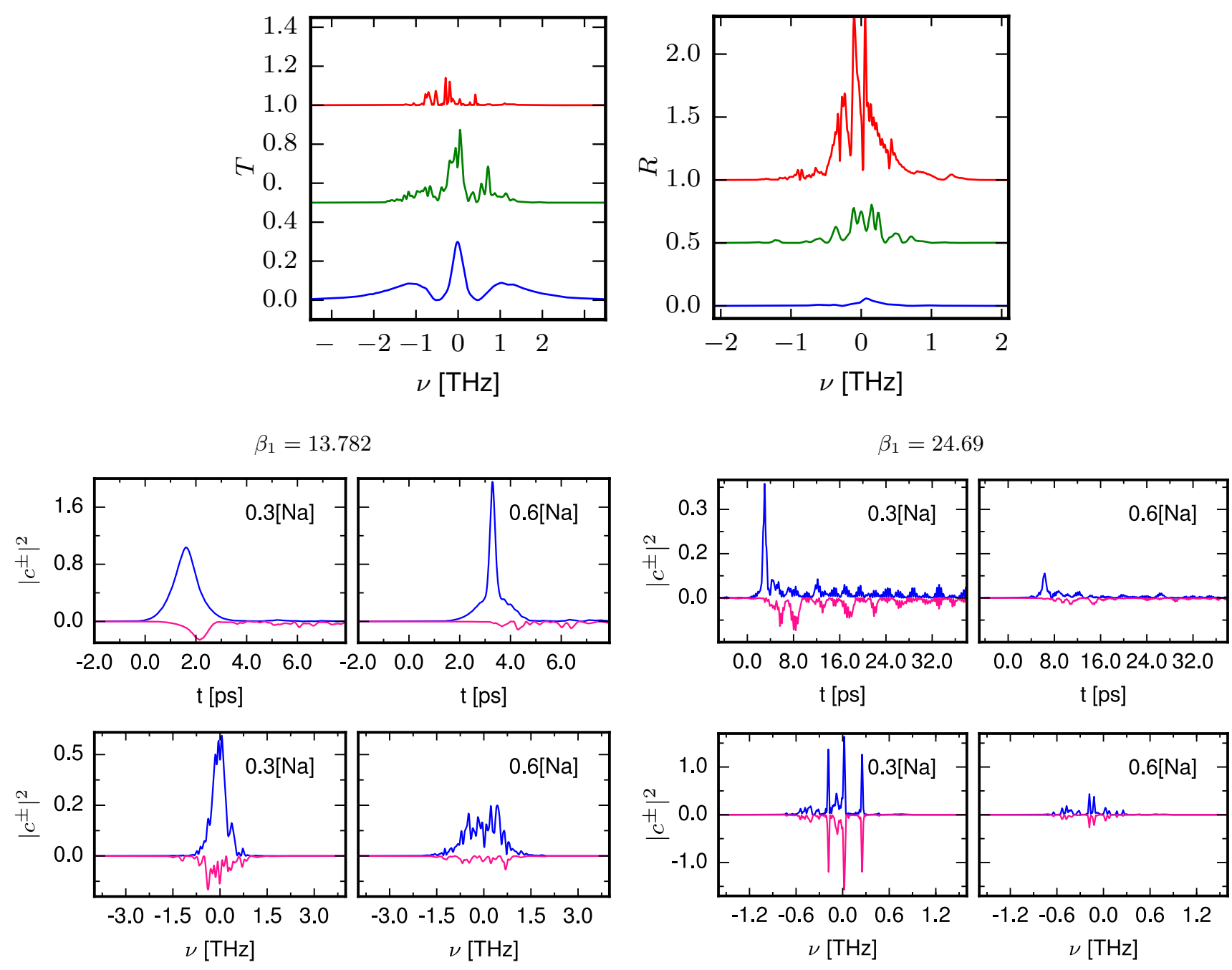

FIG. S3. (color online) The PCW is $251 a$ unit cells long, with rms roughness fixed at $0.025 a$. Transmission and reflection spectrums of an unchirped Gaussian pulse for three different group indices: 8.093(blue-bottom), 13.782(green-middle) and 24.69(red-top). Temporal and spectral profiles for the forward (blue/dark-solid) and the backward (pink/light-solid) mode envelopes at two different points inside the PCW are also shown. 